\title{
Requirement management in the European Grid Infrastructure ecosystem
}

\author{
Dr. Gergely Sipos
}

EGI.eu User Community Support

Science Park 140

1098 XG Amsterdam

The Netherlands

E-mail: gergely.sipos@egi.eu

\section{Karolis Eigelis ${ }^{1}$}

EGI.eu User Community Support

Science Park 140

1098 XG Amsterdam

The Netherlands

E-mail: karolis.eigelis@egi.eu

The evolution of the European Grid Infrastructure is driven by its users. Therefore capturing and communicating feedback from users to the infrastructure providers, technology providers and service providers is a key goal for the EGI-InSPIRE project and the EGI community as a whole. To support the evolution of the infrastructure, technology and human services of EGI, the EGI.eu organisation has defined a requirement management process and put in place software tools that support this activity within the communities. The process integrates the User Community Board, Operations Management Board and Technology Community Board with user communities, technology providers, national and international projects. Software tools, such as the EGI Requirement Tracking system (RT), RT web gadgets and wiki pages are provided for the EGI stakeholders as well as the general public to interact and monitor this process. The paper provides an overview of the EGI requirement management workflow and the tools and the achievements in gathering, analysing and addressing user community requirements since May 2010, the start of EGI.

EGI Community Forum 2012 / EMI Second Technical Conference, Munich, Germany

26-30 March, 2012

Speaker 


\section{Introduction}

The European Grid Infrastructure (EGI) is the result of pioneering work that has, over the last decade, built a collaborative production infrastructure of uniform services through the federation of national resource providers that supports multi-disciplinary science across Europe and around the world. An ecosystem of national and European funding agencies, research communities, technology providers, technology integrators, resource providers, operations centres, over 350 resource centres, coordinating bodies and other functions has now emerged to serve over 21,000 researchers in their intensive data analysis across over 15 research disciplines, carried out by over 1.4 million computing jobs a day. The EGI ecosystem provides a common foundation upon which the digital ERA can be brought online by building upon the strengths that have been developed over the last decade of deploying, operating, monitoring, accounting and supporting virtual research environments through a federation of national resource providers for multiple research communities at a European scale.

The EGI-InSPIRE project is a four year long EC-funded project that started in May 2010 to support the transition of EGI from a project-based system to a sustainable panEuropean e-infrastructure. The project established and supports Human Services (for coordination and community building), Technical Services (for supporting the interaction of user communities) and Infrastructure Services (for securely accessing resource hosted by different organizations). These services are provided by EGI.eu - the coordinating institute of EGI and EGI-InSPIRE - and by the National Grid Infrastructures (NGIs), European Intergovernmental Research Organisations (EIROs) and various national and European projects that participate in the EGI collaboration.

The evolution of the current EGI services, as well as the introduction of new services is driven by the needs coming from the researchers and infrastructure providers within EGI and the organizations they collaborate with internationally. This process is driven by a virtuous cycle that includes the prioritization of requirements, the fulfilment of these requirements by external technology providers, the assessment of the new technology releases to ensure they meet the original requirements, and then the deployment of new technology into the production infrastructure. A requirement is a request of change in a Human, Technical or Infrastructure service within EGI. By these changes EGI aims to satisfy its established user communities - from disciplines such as high energy physics, life sciences, astronomy, earth sciences, etc. - and emerging new research infrastructure communities, particularly those in ESFRI collaborations (European Strategy Forum on Research Infrastructures).

The paper describes the processes and tools that have been designed, implemented and are used by the EGI-InSPIRE project to capture, process and monitor requirements of existing and potential new user communities of EGI. These solutions facilitate 
structured interactions among three Virtual Research Communities, 200 Virtual Organisations, 350 resource centres from over 40 National Grid Infrastructures, various technology provider projects and several other technology developer and user support teams. As EGI matures the ecosystem is expected to become even more complex, but still manageable by these scalable requirement tracking tools and processes.

Section 2 provides an overview of requirement management activities in multinational distributed infrastructures and their relevance and influence on the EGI requirement tracking model. In Section 3 the EGI requirement management process is described, and the tools that are used to run it are detailed in Section 4. Section 5 provides statistics about the number of requirements that have been collected and were addressed using the process since 2010 May. The paper is closed with a summary and outline of future work in Section 6.

\section{Related works}

Documenting, analysing, prioritizing requirements, communicating them to relevant stakeholders who can implement changes are topics studied within the research field 'requirement management'. Within this field, depending on the subject entity of requirements, several definitions exist for the term 'requirement'. Consequently, various tools and processes exist to facilitate the management of these different types of requirements [20].

In an e-infrastructure ecosystem like EGI - where clients are separated from providers, where clients and providers are themselves distributed - a requirement repository can help in coordinating and tracking needs and solutions. Several tracking tools have emerged from various projects over the years and many of these are used by e-infrastructure provider communities. Nevertheless none of these collaborations are as complex as EGI, none of these tools are as open and documented as the ones that were adopted in EGI.

\subsection{Requirement tracking tools}

The Software Sustainability Institute (SSI) in the UK uses the Trac project management and issue/bug tracking tool [1] for tracking requirements. Clients of SSI are researchers who need assistance to the maintenance and sustainable operation of scientific software. The SSI Trac installation is configured to support both requirements tracking and Customer Relationship Management (CRM) needs of the institute [2]. Requirements are separated from other type of information within Trac by simply tagging tickets as "related to requirements". Classification of requirements into topics is not possible within the system. Possibility to gather common/similar requirements into topics is an important feature for EGI. 
The 'LCG Software Process \& Infrastructure' (lead by CERN) provides the Savannah issue tracking tool for projects and collaborations. The European Middleware Initiative project (EMI), the largest technology provider of EGI also uses this Savanna service to track bugs, issues, development tasks related to the middleware products that EMI delivers to e-infrastructure communities [3]. EMI tickets are separated from tickets of other projects that use the same system. Within the EMI section of Savannah developers separate requirements from software bugs or other issues using a specific field, called Category, within the tickets (see Figure 1).

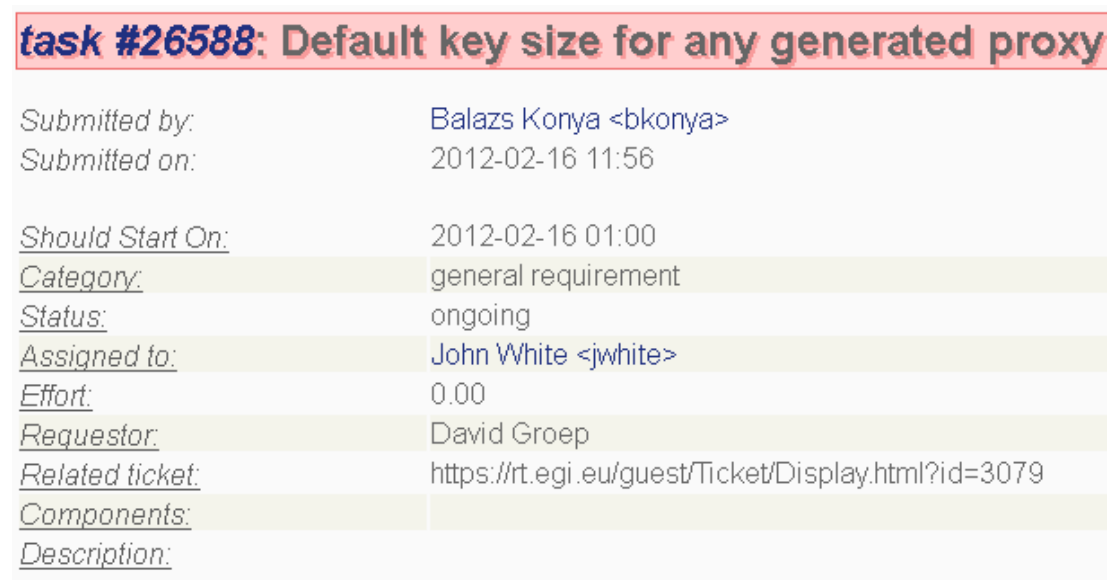

Figure 1. A requirement ticket in the EMI section of the CERN Savannah system.

'RT: Request Tracker' is an open source issue tracking tool used by thousands of organisations worldwide [4]. One of the EGI technology providers, the Initiative for Globus in Europe project (IGE) collects issues and requirements from Globus software user communities (including EGI users) in an RT installation [5]. The IGE RT is accessible in guest or in an authenticated mode. Custom fields within the tickets are used to categorise requirements by high level parameters, for example the submitter. A simple web based submission form is provided for those who would like to avoid the rather complicated web interface of RT to submit new requirements. The process by which RT is used for requirement management in IGE is described in one of the IGE deliverables (D3.2 - The IGE Requirements Management Process) [15].

The Global Grid User Support system (GGUS) [6] is a ticketing system that is developed by KIT in Germany and evolved from being a helpdesk of the WLCG project to the helpdesk of the EGEE series of projects and now helpdesk for EGI. GGUS provides a central web interface as well as regionalised interfaces (called xGUS) for users and ticket administrators who are grouped into thematic or regional 'support units'. While the system is efficient for issue management and handling, it cannot be 
used for the processing and categorisation of requirements, some of which remain open for years.

\subsection{Requirement management in e-infrastructure ecosystems}

The Extreme Science and Engineering Discovery Environment (XSEDE) [7] integrates supercomputers, high-end visualization and data analysis resources across the USA. XSEDE is a five-year project, started in 2011, co-funded by the National Science Foundation. XSEDE replaces and expands on the TeraGrid project. The XSEDE architecture extends the TeraGrid system through Operations, User services, Campus bridging and champions, Collaborative support services and Education-Outreach. According to a presentation [8] the XSEDE requirement management process is defined in a deliverable document, however the document and requirement management services are not available publicly on the XSEDE website. A feedback form is available on the XSEDE user portal [9] that invites users to provide feedback or suggestions on the further development of the portal itself, but not of other XSEDE elements.

The Open Science Grid (OSG) advances science through open distributed computing [10]. OSG is a multi-disciplinary partnership to federate local, regional, community and national cyber infrastructures in the US to meet the needs of research and academic communities at all scales. Requirement management services are not available on the project website.

The Partnership for Advanced Computing in Europe (PRACE) project provides a distributed, supercomputer-based research infrastructure [11]. The supercomputing centres of PRACE are accessible for users after a peer-reviewed application process. This process, as well as the training sessions provided for successful applicants [12], establish strong relationship between PRACE centres and users, from who PRACE collects requirements directly. Public interfaces to browse open and solved requirements, to submit new requirements is not available on the PRACE website.

\section{Requirements for an EGI requirements management system}

Research communities that already use or plan to use distributed computing infrastructures are facing various challenges which require continuous improvements and adoptions of software and services. Some of these communities - particularly high energy physics, life sciences, astronomy, earth sciences and fusion physics - developed expertise during the EGEE series of projects on how to design, develop, integrate and operate community specific applications on top of e-infrastructures. To facilitate the requirement capturing and management processes within the ecosyste, EGI.eu has setup a dedicated system for requirements tracking. Initial requirements for this system were: 
- It must be a ticket based system in which a ticket could be used to track the evolution of a requirement (the need for a new service or the need of changing an existing service).

- It must allow the clustering of related or similar tickets into topics and indicate these as concern of multiple communities.

- It must be able to cope reliably with a few hundred ticket managers and tens of thousands of ticket submitters.

- It should provide customisable interfaces for the various stakeholders, enabling them to join their own sections of the requirement management process and monitor progress within other sections.

- It should provide interfaces at different levels of complexity depending on the preference of the user.

- It must be interoperable with the ticketing systems that members of the EGI ecosystem already use to track internal tasks, issues, bugs and requirements.

- It must be embeddable into portal interfaces that members of the EGI ecosystem operate for themselves and their clients.

After the evaluation of various ticketing systems the 'RT: Request Tracker' solution has been chosen. An RT installation has been setup by the Czech NGI, (CESNET) and configured according to the requirement management process of EGI.

\section{The EGI requirement management process}

The European Grid Infrastructure emerged from the Data Grid and EGEE series of projects as a model for a sustainable e-infrastructure in Europe. The EGI model is based around strong sustainable National Grid Infrastructures that are brought together across Europe by a dedicated coordinating entity - the EGI.eu organisation. The governance of EGI.eu, a Dutch Foundation (a Stitching) is entrusted to the EGI Council which draws its membership from the national or domain specific organisational resource providers that are participants in the foundation.

A sustainable operation of EGI requires well-defined interactions with technology and infrastructure providers, as well as with the user communities to drive the evolution of various e-infrastructure services. Interactions among these groups, and among policy bodies and projects of the EGI community are established through Memorandum of Understandings (MoUs), Operational Level Agreements (OLAs) and Service Level Agreements (SLAs):

1. External technology providers wishing to deliver software into the Unified Middleware Distribution (UMD) for deployment within EGI sign an MoU to describe the main capabilities of the provided software and an SLA to define 
the various parameters for dealing with potential issues in the delivered software.

2. Structured user communities, recognised in the form of 'Virtual Research Communities' sign MoUs with EGI, describing the scientific domains that they are active in and the contact points who represent them in interacting with the various EGI functions.

3. Resource Infrastructure providers sign a specific MoU committing themselves to operational agreements and polices. This ensures that EGI's users are provided with the same quality of infrastructure regardless of who provides the particular resource/service.

4. Project MoUs are used to collaborate with EC or nationally funded projects where a mutually beneficial programme of work can be identified.

The relationship between the key groups (Technology providers, User communities, Resource providers) with the NGIs, EIROs and projects (represented through EGI.eu) is presented in a virtuous service cycle of Figure 2.

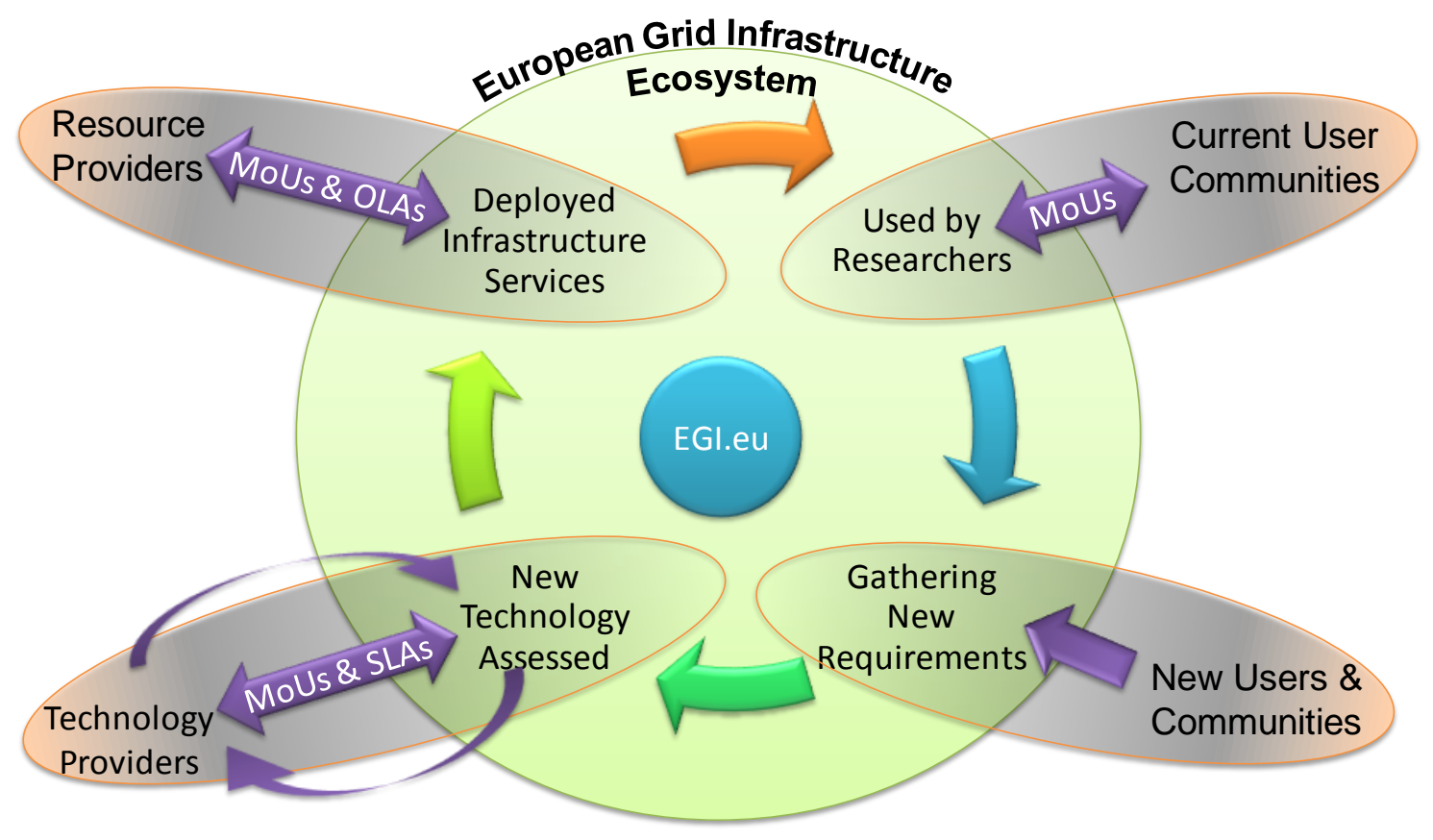

Figure 2. EGI virtuous service cycle

The EGI requirement tracking process (represented in Figure 3) is owned by the User Community Support Team of EGI.eu and operates in the following way:

1. User communities of the EGI consist of scientific users and those who provide technical support directly to them from NGIs, projects or their own communities. 
These user communities exist in EGI as VRCs, Virtual Organisations (VOs), projects or various support teams. The users/communities requirements are captured in tickets in the EGI RT system. A ticket is a request for a change in any of the human, technical or infrastructure services of EGI.

2. The User Community Support Team (UCST) of EGI.eu (through its partner from the Czech NGI) provides the RT system and performs:

- The 'normalisation' of the received requests, i.e. ensuring that the requests are complete and that the different sections of a ticket are filled consistently.

- If possible addresses the requirement and resolves the ticket. This is a typical case for requests that relate to human services and processes that are provided or owned by the team itself. (For example changes in the Training Marketplace or the Applications Database systems.)

- If the UCST cannot provide solution but assumes that its partners within the NGIs, VRCs or collaborating projects can, then UCST invites them to do so through the EGI Helpdesk. In this case a ticket is opened in the helpdesk and is linked to the RT ticket. The linkage between the tickets ensures traceability of requests across the ecosystems. Depending on the service the requirement relates to, the request may trigger the fixing of a bug in a middleware service, the reconfiguration of a software service at a site, a new development task for a technology provider, etc.

3. If the requirement cannot be addressed by any of the support teams that are present in the helpdesk then UCST ask for assistance from the User Community Board (UCB), the Technology Coordination Board (TCB) or the Operation Management Board (OMB):

- The UCB is the group of representatives from the VRCs that are using or are planning to use the production infrastructure. Through the UCB UCST can ask for clarification on the requirement or can request resources for implementing the change. This works typically for requirements that cut across multiple communities and are therefore in the common interest of multiple VRCs. For example organising workshops to collect and exchange best practices about workflow development is an activity that can be carried out by EGI in collaboration with the UCB.

- The TCB has representatives from the technology providers that are contributing software to the UMD, the middleware software that is operated on EGI resources. For UMD-related requirements the TCB can identify suitable technology providers and invite them to supply upgrades or new components into UMD in order to satisfy the request. Issues that require 
coordinated work from multiple middleware product developer teams or require significant change/addition to UMD can get addressed through the TCB. Requirements that ask for specific changes in existing UMD components are forwarded to technology providers through the EGI Helpdesk.

- The OMB has representatives from the operational staff from each of the resource providers. The OMB can help the UCST resolve issues that relate to service configurations and resource allocations. For example the setup of community-specific SLAs between sites and VRCs is a requirement that can be resolved with the assistance of the OMB.

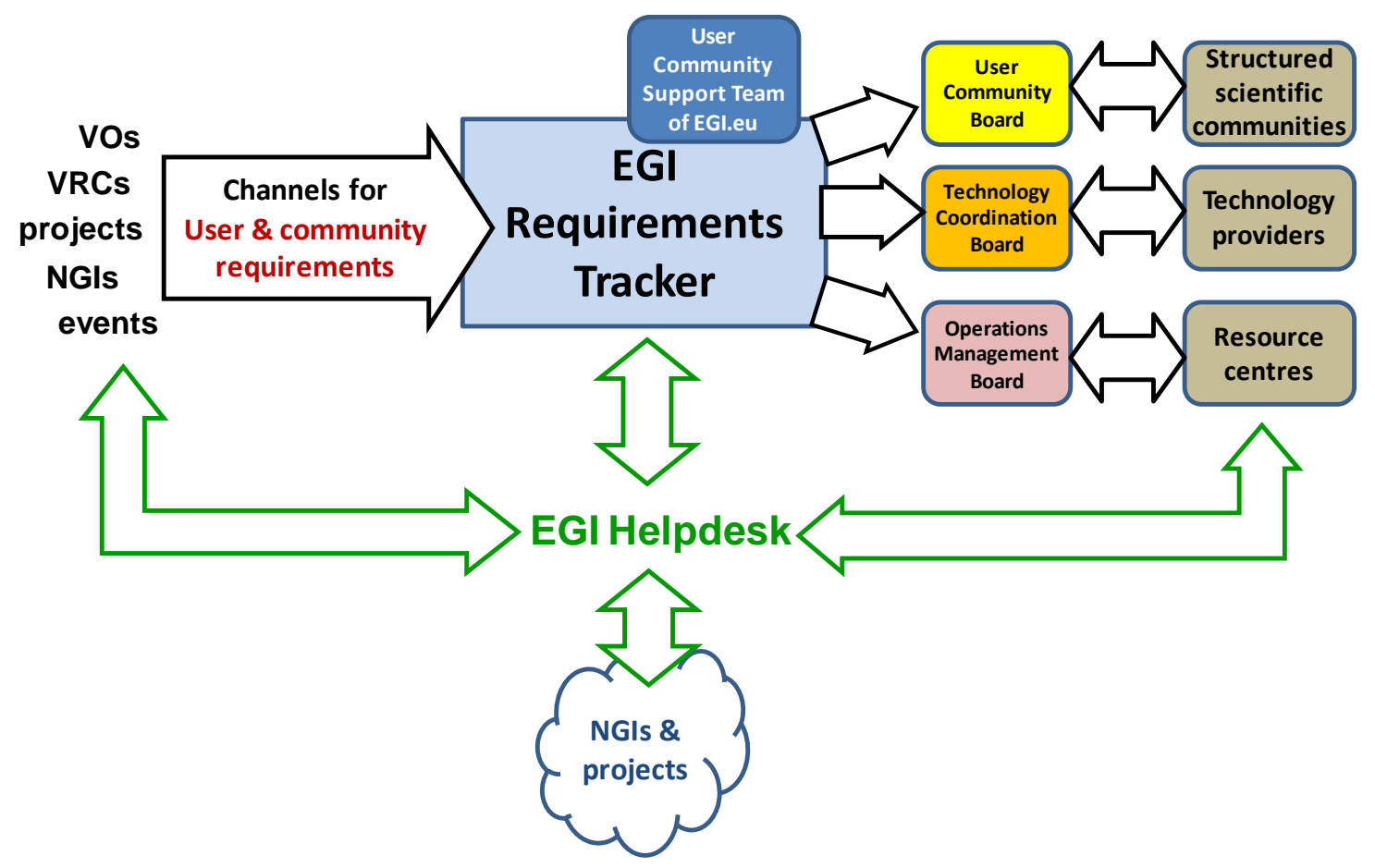

Figure 3. EGI requirements management process

\section{The EGI requirements tracker}

As it was described earlier, the main tool used by the EGI requirement management process is an EGI RT installation. RT is a highly customisable ticketing system with a web portal that ticket submitters and managers can use to interact with the system. Within the EGI RT a dedicated queue is used for the requirements. The tickets in this queue are visible without authentication to the general public [13], and are comment-able after login to the EGI Single Sign On system [16]. The tickets can be edited only by dedicated requirement engineers of EGI, primarily by members of the UCST and managers of the EGI service areas. 
The data that a requirement ticket stores initially is presented in Figure 4. Using various standard and custom fields the ticket keeps information about the requestor, provides categorisation of the request, includes the requestor's assessment of the impact of the change, a description of the requested change, indicates the status and finally the 'owner' of the requirement. After a requirement ticket is submitted, RT generates a notification to the UCST and to the owner (if filled by the requestor) so they can start the requirement processing workflow that was described in Section 4.

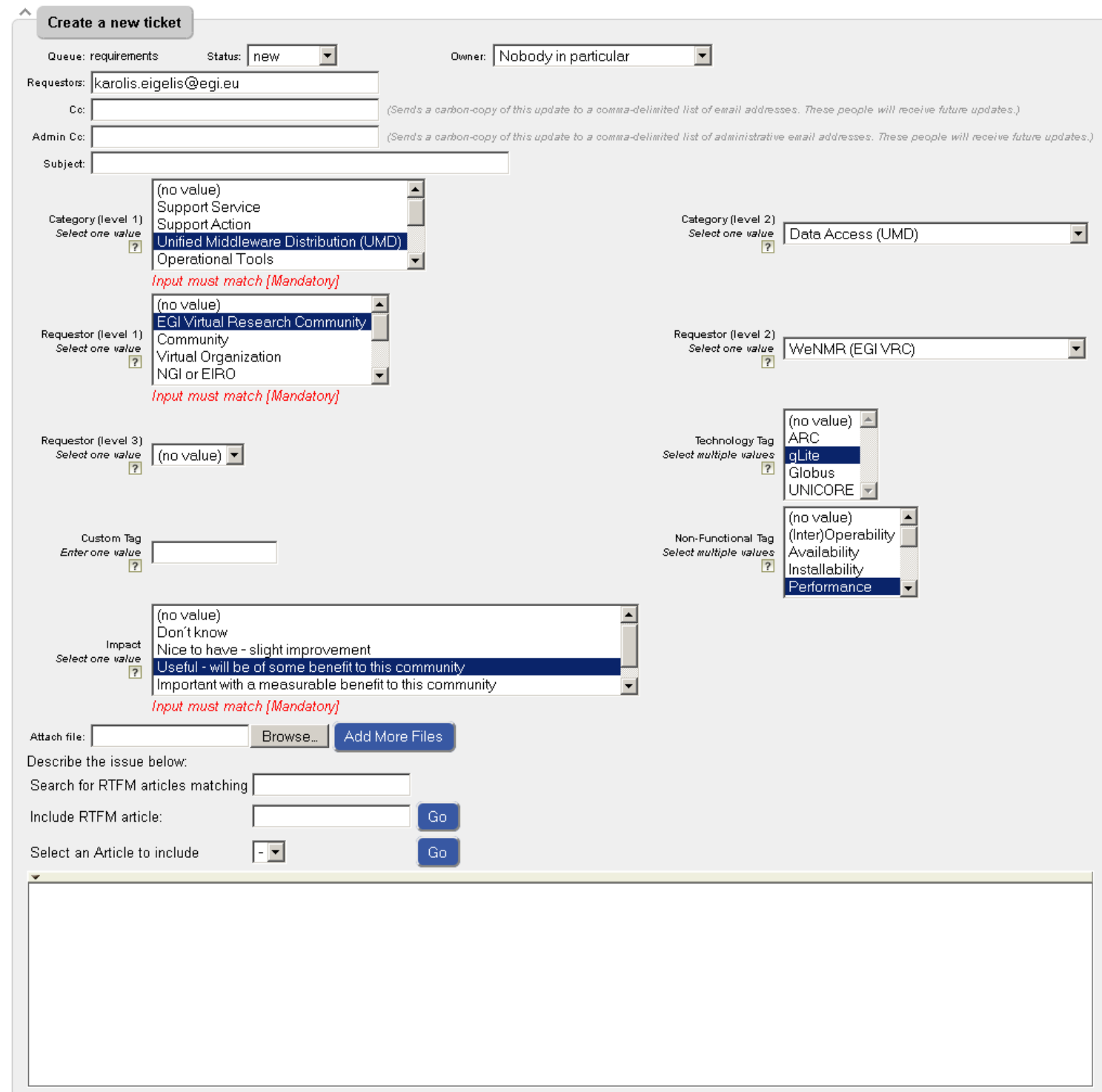

Figure 4. The requirement submission form of the EGI RT system

Different user communities, or different users from the same community may submit similar or related requirements into RT. In order to simplify the management of related items, UCST introduced the concept of 'topical tickets' in RT. Topical tickets 
act as boxes that store related tickets. Topical tickets give emphasis to important issues because they indicate that a change would bring benefits to multiple people or to multiple communities.

RT provides web interfaces to browse, search and list requirement tickets. Some of these interfaces can be customised and turned into 'personal dashboards' to present those tickets and action buttons that are relevant for a specific user. For example members of a VRC, a VO, an NGI or a project can list the requirements they submitted to EGI, or requirements that they own (need to respond to). A dashboard editor is provided by RT for users to define the custom parameters of their dashboards (See Figure 5).
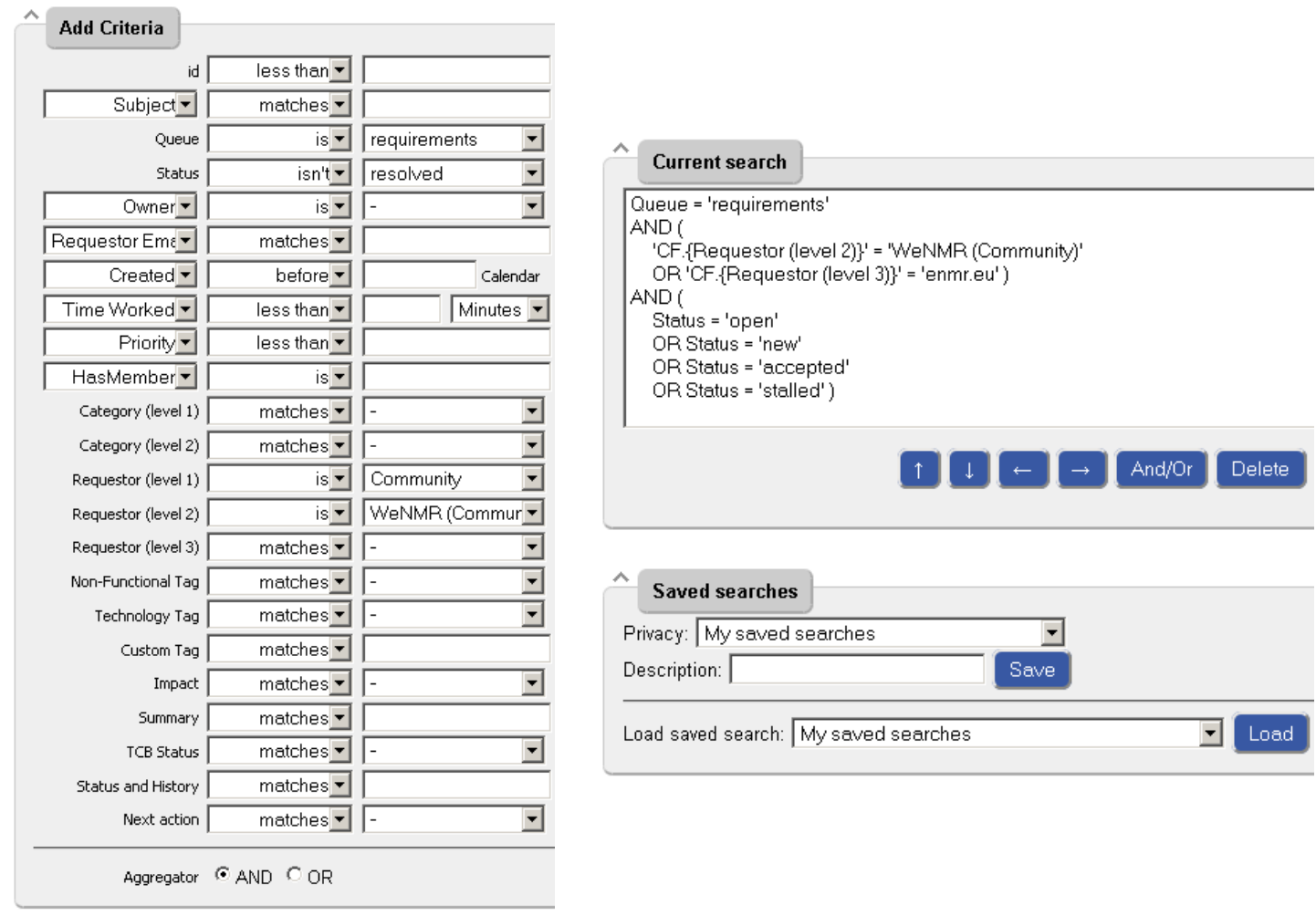

1

Figure 5.Configuration interfaces to create personalised requirements dashboards

Although RT is highly configurable, its web interfaces - including the personalised dashboards - have limitations in expressing the status of a requirement within the requirements management workflow that was described in Section 4. To overcome this limitation the UCST has setup an extra interface for RT. The interface is a publicly accessible site within the EGI mediawiki installation [13] and provides an intuitive, coloured list of requirements. The different colours represent the different stages of the processing workflow so the requirements on the page are coloured according to their position within the workflow (See Figure 6). The information on this page is 
automatically updated when certain fields within the ticket change. The synchronisation between RT tickets and the Wiki page are done with a mediawiki extension, called 'Rtreader'. Rtreader simplifies requirement administration and results a simple, yet informative and robust interface for EGI members for the general public about EGI user community requirements.

\section{Explanation of colour codes used below}

Orange indicates that the Technology Coordination Board, or technology providers in the board follow up the requirement. Green indicates that technology providers follow up the requirement through the EGI Helpdesk.

Blue indicates that EGI.eu with the $\mathrm{NGI}$ support teams follow up the requirement.

Yellow indicates that the requirement needs input/decision from the User Community Board

Pink indicates that the requirement needs input/decision from the Operations Management Board

\begin{tabular}{|c|c|}
\hline $\begin{array}{l}\text { \#2968 JDL does not offer } \\
\text { GLUE attribute for } \\
\text { available disk space on } \\
\text { worker node } \\
\text { BUG / Fixed and } \\
\text { released in EMI-2 }\end{array}$ & $\begin{array}{l}\text { 2012, August 13: Requestor was informed about the implemented requirement in UMD-2.1.0 release and was } \\
\text { asked about a feedback within next } 2 \text { weeks. } \\
\text { 2012, August 6: UMD-2.1.0 was released and includes fixed bug. } \\
\text { 2012, July 25: Unfortunately UMD-2 release did not include updates from EMI-2 due to globus libraries issues. } \\
\text { Promised to be release in UMD-2.0.1 } \\
\text { 2012, May 24: UCST informed requirement owner about the improvements and asked for a feedback. } \\
\text { 2012, May 21: EMI-2 was released including the bug fix. } \\
\text { 2011, October 14: savannah bug was created. REF \#87799 } \mathrm{i} \\
\text { 2011, October 14: requirement is at GGUS. REF: \#75297 } \\
\text { 2011, February 7: New requirement entered into RT }\end{array}$ \\
\hline $\begin{array}{l}\text { \#2985 VO registration } \\
\text { without certificates } 1\end{array}$ & 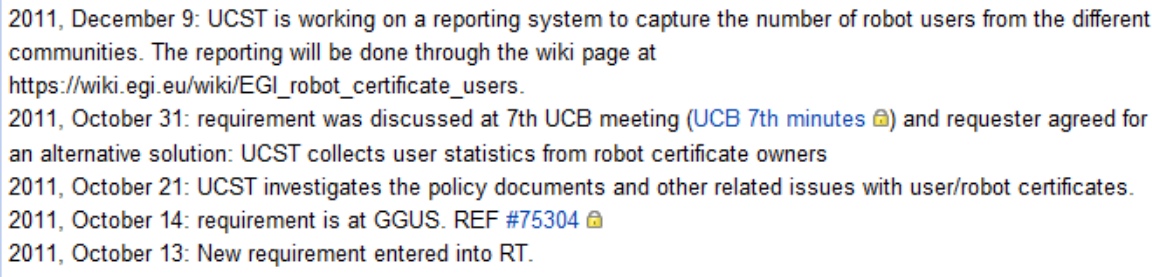 \\
\hline $\begin{array}{l}\# 3070 \text { NGls publishing } \\
\text { user-level accounting } \\
\text { data to the authorised } \\
\text { VO Resource } \\
\text { Managers }\end{array}$ & $\begin{array}{l}\text { 2011, November 22: operations team are working on a wide NGI-VO SLA document } \mathrm{E} \\
\text { 2011, October 31: requestor was asked to provide more details. } \\
\text { 2011, October 31: requirement is under evaluation by UCST. } \\
\text { 2011, October 27: New requirement entered into RT. } \\
\text { 2011, October 26: requirement is at GGUS. REF: \#75762 - }\end{array}$ \\
\hline $\begin{array}{l}\text { \#2947 EMI/UMD grid } \\
\text { elements available as } \\
\text { virtual machine images } \\
\text { TASK } / \text { ? }\end{array}$ & $\begin{array}{l}\text { 2011, October 3: requirement already has savannah Task. REF: \#22854 } \mathrm{A} \\
\text { 2011, October 3: New requirement entered into RT. }\end{array}$ \\
\hline $\begin{array}{l}\text { \#3563 Full support for } \\
\text { PCKS8 private keys } \\
\text { BUG / Fixed }\end{array}$ & $\begin{array}{l}2012 \text { March 21: this requirement will be submitted to TCB in April } \\
2012 \text { March 21: Bug was fixed REF: globus_gsi_credential-3.7.tar.gz } \\
2012 \text { March 21: new requirement }\end{array}$ \\
\hline
\end{tabular}

Figure 6.Colour coded listing of EGI user community requirements on a public wiki page [13]. 
Another issue that we faced at an early stage of EGI was that many of the EGI stakeholders, especially scientific groups had already built communities around web portals that integrate relevant applications, services and data from various sources. The users of such portals are less willing to leave the site they are using to visit another one and access services there. They expect interfaces for requirement management to be part of their well-known and trusted portal environments. To make EGI requirements embeddable into such websites our team has developed two web gadgets for the EGI RT system [14].

Web gadgets (or widgets) are part of a rather broad concept, based on the general idea of code reuse, targeted towards the reuse of components within Web pages. A web gadget is a small application that can be embedded into third party web sites by any user on a page where they have rights of authorship (for example within a user's personal website, within an NGI or a VRC website). The 'Requirements tracker gadget' [13] can present user/community specific requirements within a website; the 'Requirement submission gadget' provides a simple form to submit requirements to EGI from any site. Both gadgets can be customised from the functional and the presentation perspectives (See Figure 7). 
WeNMR Virtual Research Community Open Requirements

\#3588 Re: [VOMS Admin] User suspension notification

by Alexandre Bonvin - Apr 02, 2012

Dear all In case you have not seen it yet, check the blog I...

\#3588 Re: VOMS Adminl User suspension notification

\#3446 implementinq per-site vo adm view

\#3070 NGls publishing user-level accounting data to the authorised vo Resource Managers

\#2985 Vo reqistration without certificates

\#2947 EMMIUMD qrid elements available as virtual machine images

\#2877 storaqe element and lifetime of data

$\# 1742$ Ica-cp - backaround replication to other SES (WeNMR)

\#1550 Graphical configuration tool for qLite

Show Solved Requirements

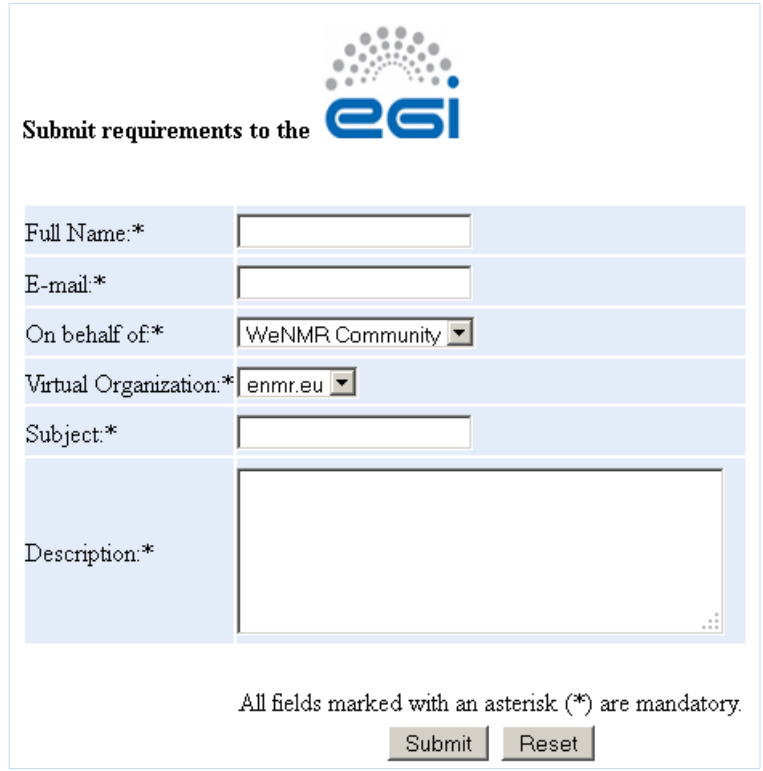

Figure 7. WeNMR VRC specific instances of the EGI requirement tracker and requirement submission web gadgets.

\section{Requirement statistics}

Using the native reporting tool of RT we can generate detailed statistics about the requirements that are at the different stages of the processing workflow. The data can even be exported from RT in CVS format. Since the start of EGI (May 2010) until the time of writing (April 2012) the EGI community captured 546 requirements: 251 requests from user communities, 295 requests from site administrators. 131 out of the 251 user community tickets are already solved, 120 are still open at various stages in the workflow. 63 out of the 120 open requirements are high level needs of ESFRI projects and were gathered from various high level documents, such as the report, titled 'ESFRI project requirements for Pan-European e-infrastructure', from the European E-infrastructures Forum [17]. The key statistics about requirements stored in the EGI RT system can be seen in Figure 8. 


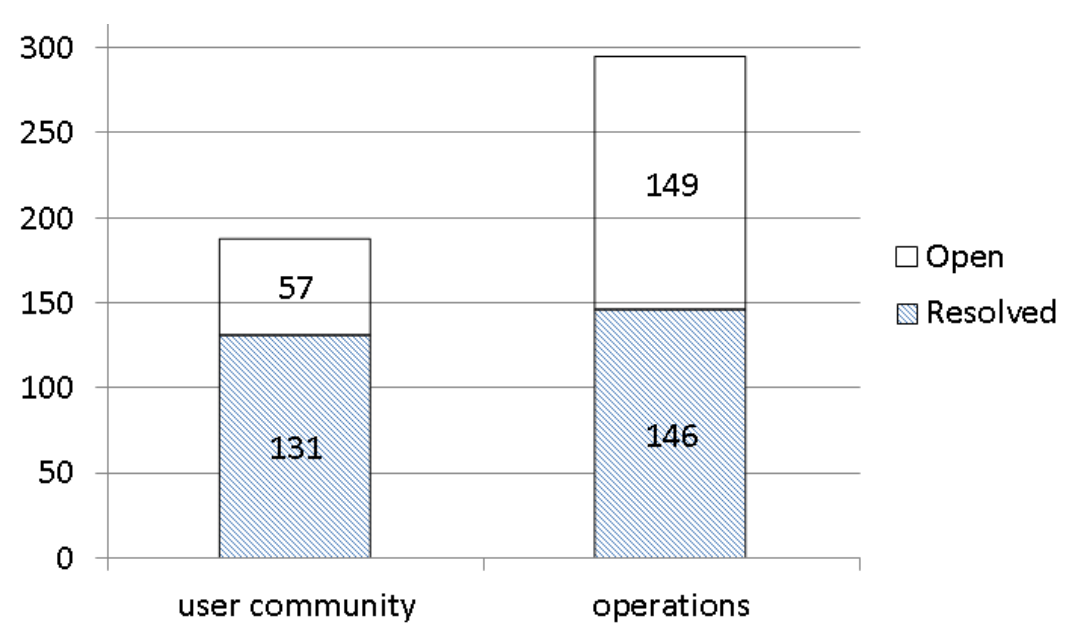

Figure 8. The status of EGI community requirements (Data taken: 23 April, 2012).

In terms of most active submitters, the highest number of requirements originate from the Life Sciences Grid Community VRC (22), followed by the WeNMR VRC (19) and the WLCG VRC (12). Out of the 546 requirements 32 requirements asked for improvement/change in the UMD, the spread of these across the UMD components can be seen in Figure 9.

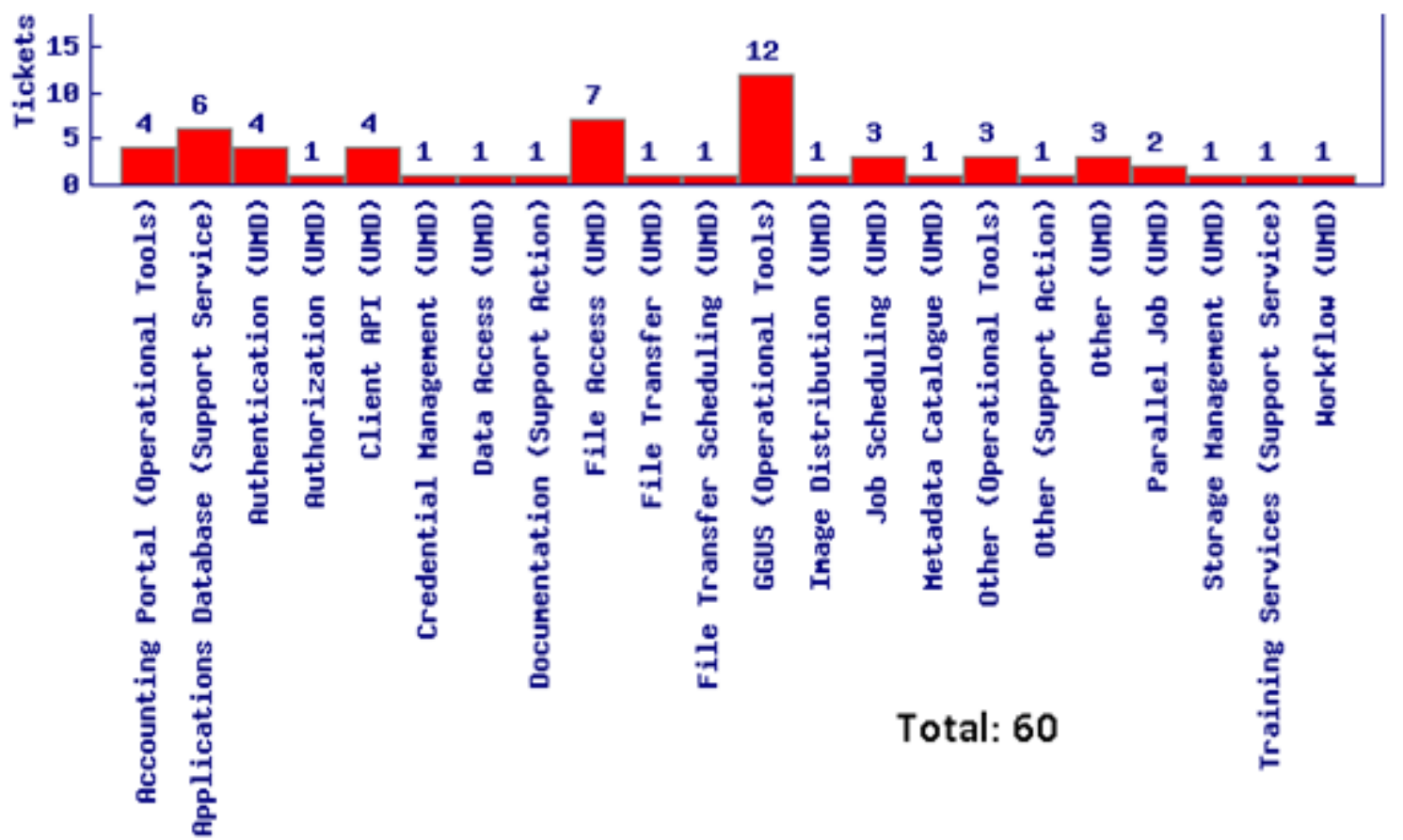

Figure 9. The distribution of user requirements across software tools/services in and outside of UMD for which the requirements requested changes/updates. 


\section{Summary and future work}

The EGI User Community Support and Operation teams have put in place a robust process to capture, analyse and address user requirements. The process respects the distributed, federated nature of the infrastructure, its user, operational and technology developer communities. The workflow enables the integration of new solutions in the ecosystem and facilitates the reuse of best practices, tools services across resource centres and scientific disciplines.

Through the requirement workflow the various support and developer teams solved 277 requirements across the user, operations and technology domains in less than two years. Some of the complex requirements that cut across multiple user communities can be solved only through extensive consultation with these communities, with software and service providers. Examples of such topics are high level, integrated services and APIs to interact with middleware services and federated identity management to access infrastructure services. Investigations in these areas are on-going using the EGI Helpdesk, surveys and through topical workshops.

The EGI RT system and its related tools - the requirements table in the Wiki and the RT gadgets - provide generic and personalised interfaces to interact with the requirement tracker. These tools keep requestors informed about progress and next actions, without overloading them information. Personalised, NGI and community specific dashboards and gadgets are already used in two communities: Life Sciences Grid Community and WeNMR VRCs. RT dashboards are preferred by NGIs. Besides promoting the customisable interfaces to EGI members, the UCST will also investigate the feasibility of providing mobile applications (primarily Android apps) for the RT system, and for other user support services that come from the EGI-InSPIRE project (Training Marketplace, Applications Database, Client Relationship Manager).

\section{Acknowledgement}

The work presented in this paper is supported by the EGI-InSPIRE project (Integrated Sustainable Pan-European Infrastructure for Researchers in Europe), cofunded by the European Commission (contract number: RI-261323) for four years from the $1^{\text {st }}$ of May 2010. EGI-InSPIRE is a collaborative effort involving more than 50 institutions in over 40 countries. Its mission is to establish a sustainable European Grid Infrastructure (EGI).

\section{References}

[1] Trac tool: http://trac.edgewall.org/

[2] Rob Baxter, Neil Chue Hong, Tracking community intelligence with Trac, Philosophical Transactions, Royal Society, Volume 369, No 1949, pp 3372-3383, 2011. 
[3] EMI requirements group based on Savannah: https://savannah.cern.ch/task/?group=emi-req

[4] RT: Request Tracker: http://bestpractical.com/rt/

[5] IGE requirements submit form, http://www.ige-project.eu/hub/rt/submit

[6] T Antoni, W Bühler, H Dres, G Grein and M Roth, Global Grid User Support - Building a worldwide distributed user support infrastructure, Journal of Physics: Conference Series, Volume 119, Part 5, 2002.

[7] Publication by XSEDE, What is XSEDE?, https://xup1.tacc.utexas.edu:8443/documents/18157/169845/what-is-XSEDE.pdf

[8] Presentation by John Towns, XSEDE Overview, DEISA-PRACE Symposium, Helsinki, Finland 2011 April.

[9] XSEDE feedback form, https://tis.xsede.org/feedback

[10] Building and Testing a Production Quality Grid Software Distribution for Open Science Grid, Alain Roy, et. al. In Journal of Physics: Conference Series, SciDAC 2009, Volume 180, 012052, 2009. Edited by Horst Simon.

[11] PRACE general presentation, "Partnership For Advanced Computing In Europe", http://www.prace-ri.eu/IMG/pdf/prace-general-presentation-2010-2.pdf

[12] Presentation by Tim Stitt and Tim Robinson, "Training Survey", www.praceproject.eu/IMG/pdf/PRACE-TrainingSurvey-2.pdf

[13] Overview of EGI user community requirements: http://go.egi.eu/requirements

[14] Web gadgets for EGI requirement management, http://www.egi.eu/services/support/gadgets/rt/index.html

[15] Initiative for Globus in Europe project: The IGE Requirements Management Process: https://docs.google.com/viewer?a=v\&pid=sites\&srcid=aWdlLXByb2plY3QuZXV8cHVibGljfGd4O jY4YWJhZWIxYTJjYzZjNg

[16] EGI Single Sign On system: http://www.egi.eu/sso

[17] European E-Infrastructure Forum: ESFRI project requirements for Pan-European e-infrastructure resources and facilities, 2010, April 28, available online at https://documents.egi.eu/document/12 